\title{
Hybrid arch frozen elephant trunk repair for acute type $A$ intramural hematoma
}

\author{
Fadi Hage", Ali Hage", Michael W. A. Chu \\ Division of Cardiac Surgery, Department of Surgery, Western University, London Health Sciences Centre, London, ON, Canada \\ \#These authors contributed equally to this work and are co-first authors. \\ Correspondence to: Dr. Michael W. A. Chu, MD, FRCSC. Professor of Surgery, B6-106 University Hospital, LHSC, 339 Windermere Road, London, \\ Ontario, N6A 5A5, Canada. Email: michael.chu@lhsc.on.ca.
}

Submitted Jun 28, 2019. Accepted for publication: Jul 25, 2019.

doi: 10.21037/acs.2019.08.01

View this article at: http://dx.doi.org/10.21037/acs.2019.08.01

\section{Introduction}

Contemporary techniques for surgical repair of acute type A aortic dissection and intramural hematoma (IMH) have recently expanded to include conventional, endovascular and hybrid options. The hybrid arch frozen elephant trunk (FET) repair provides an attractive option to treat patients with more extensive multisegment aortic involvement with aggressive distal remodelling in a single-stage operation (1). We believe the FET promotes true lumen expansion, false lumen collapse with a potentially reduced risk of late aneurysm formation and need for distal aortic operation (2-4). This strategy is also less affected by the challenges of current endovascular arch techniques such the unstable or challenging proximal seal zone, higher stroke rates, long-term durability and limited suitable patient anatomy $(5,6)$.

Off-label use of conventional thoracic endografts as FET is being increasing supplanted by commercially available hybrid arch graft designs. The multibranched Thoraflex hybrid graft has a robust proximal arch Dacron collar which nearly eliminates the risk of type $1 \mathrm{~A}$ endoleaks $(7,8)$ and helps to achieve optimal hemostasis in challenging IMH cases. Additionally, the unique distal nitinol ringed stent design allows the FET graft to sit well within the distal arch and proximal descending aorta, including tortuous or calcified anatomy that can accompany patients with acute IMH (9). Lastly, the multiple device branches can simplify head vessel branch reconstruction while shortening circulatory arrest and overall operative times.

\section{Clinical vignette}

A 61-year-old male presented with sudden tearing chest and back pain with spontaneous loss of vision in both eyes. These symptoms improved after several hours and the patient did not seek medical attention until 36 hours later when he had persistent headaches. In the Emergency Room, he was profoundly hypertensive with systolic blood pressures $>200 \mathrm{mmHg}$. Unfortunately, he was discharged home on multiple antihypertensive medications and brought back for an urgent CT scan 48 hours later. Computed tomography (Figure 1) demonstrated an acute type A IMH extending from the sinotubular junction, through the ascending aorta and arch to the proximal descending aorta. He was also found to have an aneurysmal aortic arch with possible contained rupture/ pseudoaneurysm or a penetrating aortic ulcer. Preoperative transthoracic echocardiogram revealed normal biventricular size and function with no significant valve abnormalities. The aortic root was normal in size and measured $32 \mathrm{~mm}$ at the sinuses of Valsalva. The proximal ascending aorta measured $42 \mathrm{~mm}$ and the aortic arch $47 \mathrm{~mm}$ with a suggestion of flow in the region of the suspected contained rupture in the arch. Coronary angiography revealed significant left anterior descending and obtuse marginal artery disease. The patient's past medical history was significant for recalcitrant hypertension, hyperlipidemia, previous smoking, obstructive sleep apnea, bilateral inguinal hernia repairs, and an umbilical hernia.

Based on the patient's stability, subacute presentation 

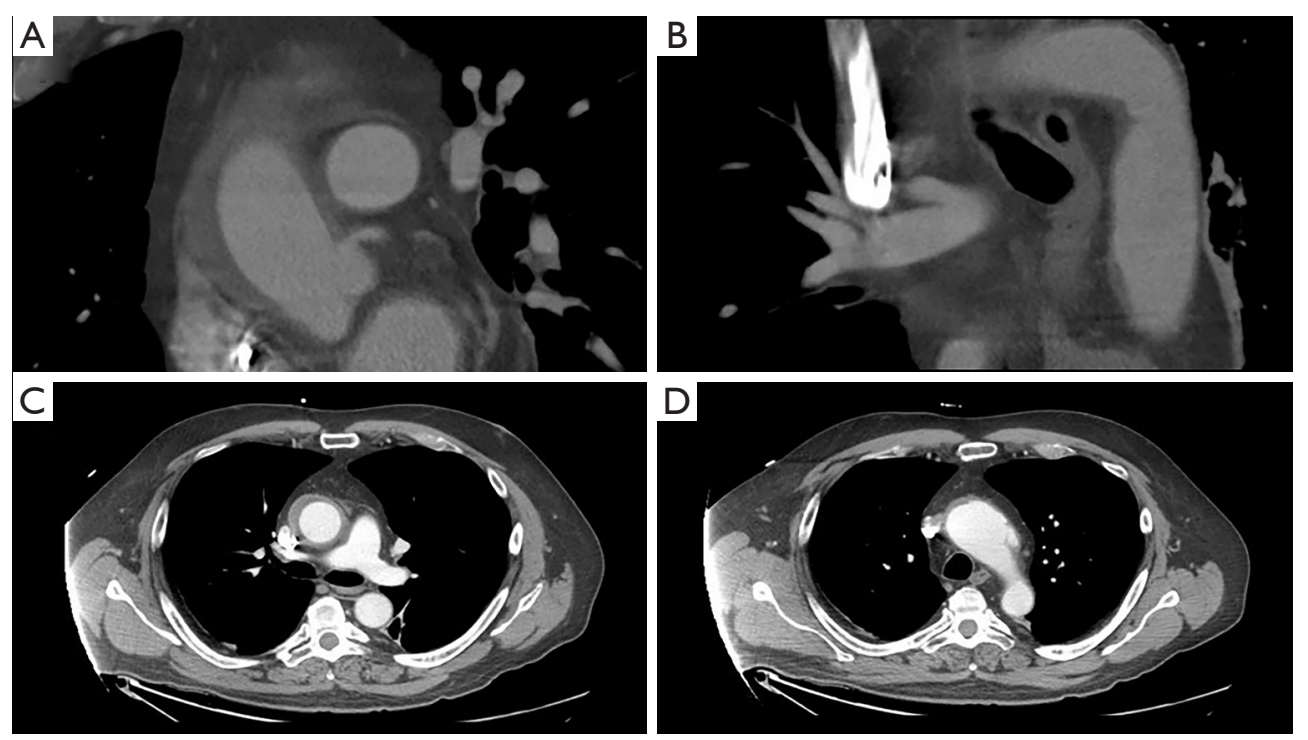

Figure 1 Preoperative computed tomography of the aorta showing an acute type A intramural hematoma extending from the sinotubular junction, through the ascending aorta $(\mathrm{A}, \mathrm{C})$ and arch to the proximal descending aorta (B). It also shows an aneurysmal aortic arch with possible contained rupture or a penetrating aortic ulcer (D).

and the deep positioning of the left subclavian artery, we elected to first perform a preoperative left carotidsubclavian bypass with subclavian ligation to assist with the deeply positioned subclavian ostium (10). The patient was subsequently brought to the hybrid operating room where we describe the hybrid arch and FET reconstruction.

\section{Surgical techniques}

\section{Preparation and cannulation}

The procedure was performed under general anesthesia with transesophageal echocardiography (TEE) guidance in a hybrid operating room. The patient was monitored with radial and femoral arterial lines and bilateral cerebral and lower limb oximetry. A $4 \mathrm{~cm}$ right infraclavicular incision was made to expose the right axillary artery where an 8-mm Dacron graft was sewn in an end-to-side fashion for initiation of cardiopulmonary bypass. Using ultrasound guidance, a 6-French sheath was placed in the right common femoral artery. A guidewire and Judkin's Right 4 catheter were advanced up into the aortic arch under transesophageal echocardiographic visualization, taking care to avoid the area of contained rupture with pseudoaneurysm. We then performed a standard midline sternotomy and the coronary bypass conduits were harvested. After systemic heparinization, cardiopulmonary bypass was initiated with central cooling to $25^{\circ} \mathrm{C}$ via standard right atrial venous cannulation.

\section{Aortic exposure, cross-clamp, and distal coronary artery bypass graft (CABG) anastomoses}

We skeletonized the aortic root, ascending aorta and aortic arch, gently isolating the innominate and carotid vessels. There were dense adhesions likely related to the recent acute rupture. We then identified our distal targets to be the left anterior descending artery and the obtuse marginal artery which were heavily diseased. The aortic cross clamp was carefully applied and a transverse aortotomy was made through the IMH, allowing for the delivery of cold Del Nido cardioplegia through the coronary artery ostia (Figure 2A). We constructed the vein graft bypass to the obtuse marginal and the left internal thoracic artery to left anterior descending artery bypass. For the remainder of the case, we gave intermittent antegrade cardioplegia through the saphenous vein graft and coronary ostial catheters.

\section{Proximal aortic anastomosis}

We carefully removed the IMH from the ascending aortic wall, being sure not to leave any mobile components with 

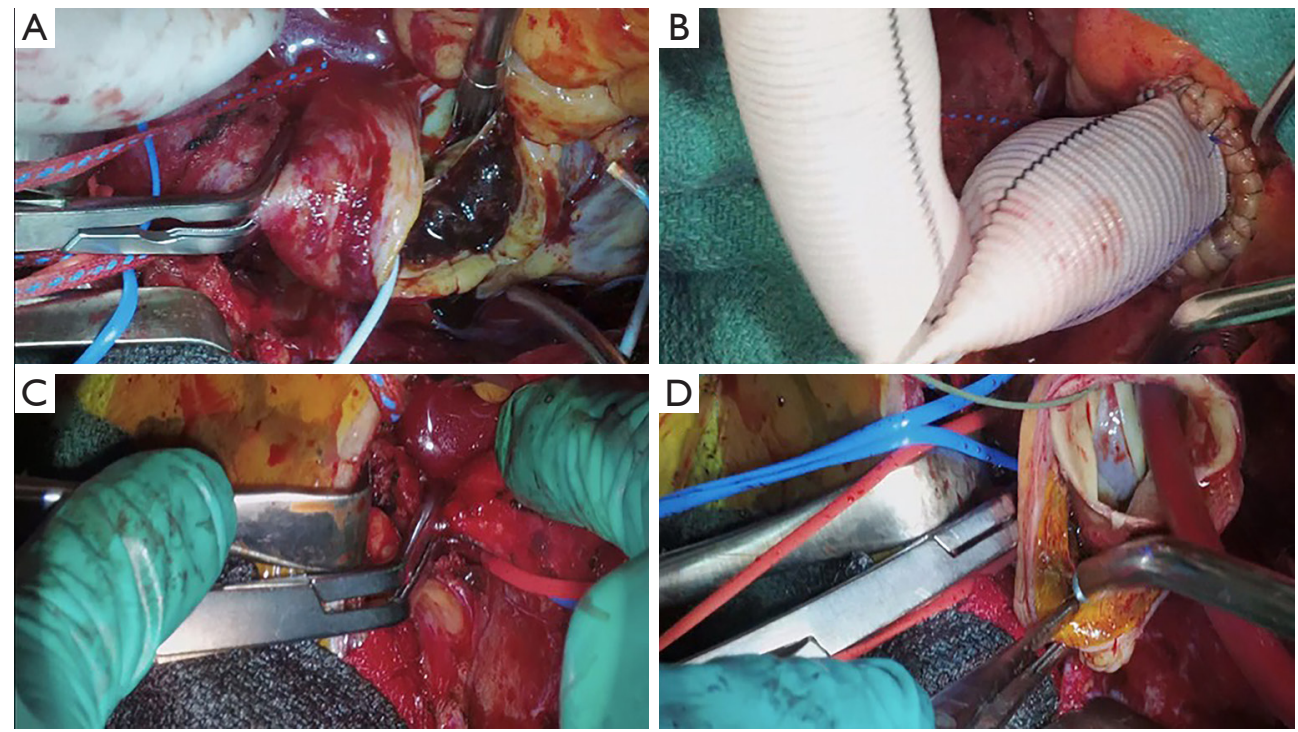

Figure 2 Surgical steps for proximal aortic anastomosis and circulatory arrest. (A) Transverse aortotomy exposing the intramural hematoma; (B) resection of the ascending aorta down to the sinotubular junction and reconstruction with a telescoping end-to-end anastomosis; (C) clamping of base of the common innominate/left common carotid trunk; (D) initiation of circulatory arrest.

in the aortic lumen which would pose a major risk for embolization and stroke. We then evaluated the integrity of the aortic valve and root and determined that the tricuspid aortic valve was functioning well with pliable cusps and there were no significant IMH or aneurysmal changes in the aortic root. As such, we resected the ascending aorta to the level of the sinotubular junction and sewed a $28 \mathrm{~mm}$ Dacron graft in a telescoping end-to-end fashion using running 4-0 Prolene (Figure 2B).

\section{Circulatory arrest and aortic resection}

After reaching a nadir temperature of $25^{\circ} \mathrm{C}$, we initiated circulatory arrest by decreasing flows to 1.5 liters per minute through the right axillary artery and placed a clamp at the base of the common innominate and left common carotid trunk (bovine arch; Figure 2C,D). We then resected the involved ascending aorta and proximal aortic arch to prepare for a zone 2 anastamosis. We could observe the area where the aorta had ruptured and the associated pseudoaneurysm which we also resected; however, there remained a very thinned aortic wall on the lesser curve of the zone 2 arch to perform the anastomosis. We feel that the Thoraflex collar and distal FET greatly enhance the hemostasis and security of this anastomosis particularly when the aortic wall is fragile as in an acute type A aortic dissection or acute IMH.

\section{Distal aortic anastomosis and Thoraflex graft deployment}

We placed 2-0 Ethibond sutures in zone 2 radially along the aortic circumference (Figure $3 A$ ). The exposure was quite challenging as the aortic arch was positioned very deep in the mediastinum at that level. Subsequently, we selected a $28 \mathrm{~mm} \times 30 \mathrm{~mm} \times 100 \mathrm{~mm}$ Thoraflex graft. We passed an Amplatz extrastiff wire through the previously placed JR4 diagnostic catheter into the mediastinum to gain through and through access (Figure 3B). Using a body floss technique for stabilization, we passed the Thoraflex hybrid graft along the guidewire through the zone 2 arch deep into the descending aorta and deployed the graft without difficulty (Figure 3C). Next, we passed the interrupted Ethibond sutures through the sewing collar in the Thoraflex graft and tied the sutures down (Figure 3D). We then ran a second layer of 2-0 Prolene for additional hemostasis. A cross-clamp was placed in the body of the Thoraflex graft between the perfusion limb and carotid limb to enable early lower body reperfusion through the Thoraflex side limb at $30{ }^{\circ} \mathrm{C}$. We constructed the carotid limb directly to the left common carotid artery using running 6-0 Prolene suture and the clamp was moved more proximally in the arch graft 

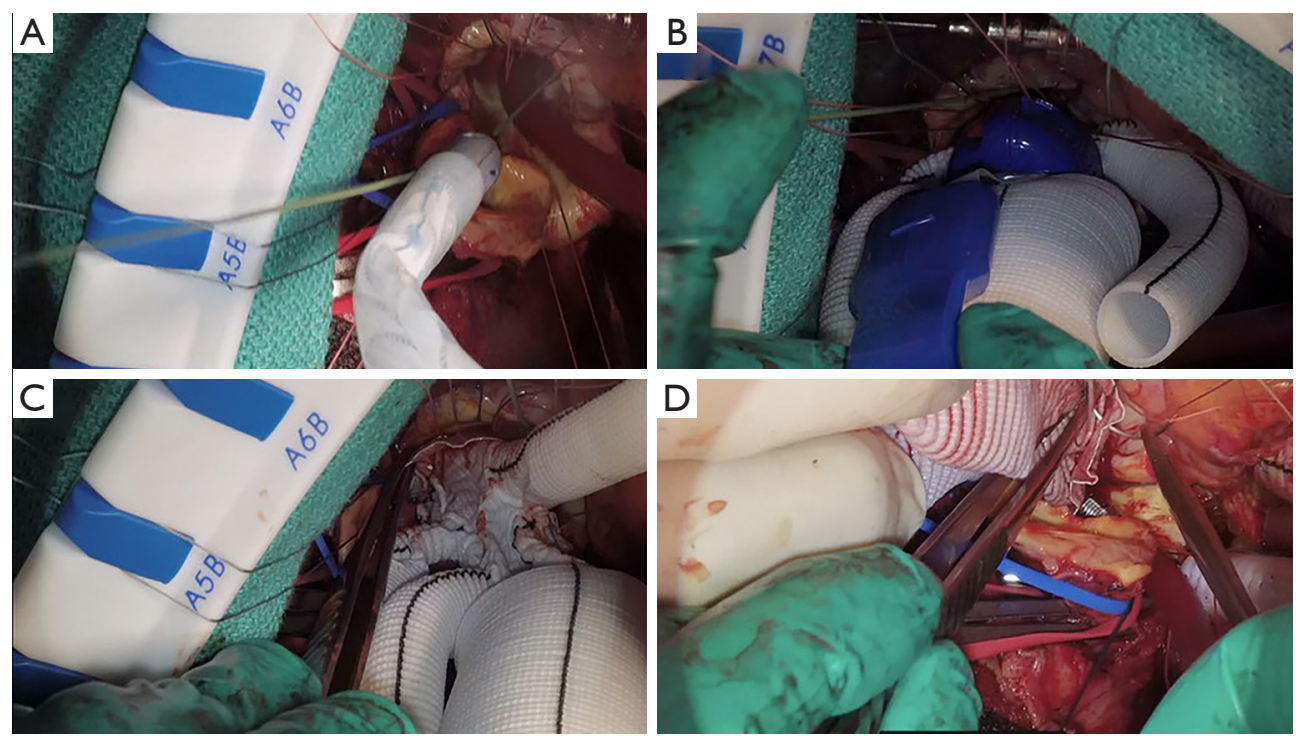

Figure 3 Surgical steps for distal aortic anastomosis and Thoraflex graft deployment. (A) Placement of interrupted Ethibond sutures in zone 2 and advancement of the Thoraflex graft over the Amplatz extrastiff wire; (B) positioning of the Thoraflex graft with correct orientation of the side limbs; (C) deployment of the graft; (D) passing of the Ethibond sutures through the sewing collar in the Thoraflex graft.

to allow antegrade bilateral cerebral flow (Figure 4A).

\section{Arch and epiaortic vessel completion}

We turned our attention back to the heart and ascending aorta where we anastamosed the ascending aortic graft to the arch graft with 5-0 prolene, tailoring the lesser and greater curves of the aorta to encourage laminar flow (Figure 4B). We completed the proximal anastomosis for the vein graft, and the cross clamp was removed to allow cardiac reperfusion. We then completed the head vessel reconstruction by anastomosing the innominate limb to the innominate artery using 5-0 Prolene (Figure 4C). We prefer to keep the epiaortic branches of the Thoraflex hybrid graft as short as possible to minimize any risk of kinking or redundancy (Figure 4D).

\section{Weaning from cardiopulmonary bypass and chest closure}

After a period of reperfusion, we weaned the patient from cardiopulmonary bypass without inotropes and only low dose vasopressor support. Protamine sulfate was administered in the normal fashion and the right axillary and thoraflex perfusion side-limb grafts were transected. The axillary graft stump is intentionally left a little longer with heavy silk ties and clips at the base in case reoperative arch surgery is required in the future. The upper pericardium is generally pulled together to reduce the risk of graft adherence to the sternum, particularly if there is limited mediastinal space, extra-anatomic bypasses to the head vessels (most commonly left subclavian) or the arch or ascending aortic grafts are inadequately shortened.

\section{Postoperative outcome}

Postoperative transesophageal echo demonstrated excellent aortic valve function with no aortic insufficiency, no gradient across the valve, and preserved biventricular function. The FET was well seated in the descending aorta. It appeared fully deployed without any signs of endoleak. The patient remained hemodynamically stable and off inotropic support before being transferred back to the intensive care unit. He was extubated on the evening of surgery, and transferred to the ward on the first postoperative day, where he recovered well without any complications allowing for his discharge home on the fourth postoperative day. A transthoracic echocardiogram was performed prior to discharge, showing a well-functioning aortic valve with no aortic insufficiency and normal left ventricular function with an ejection fraction of $55 \%$ to $60 \%$. A CT scan was also performed, which demonstrated 

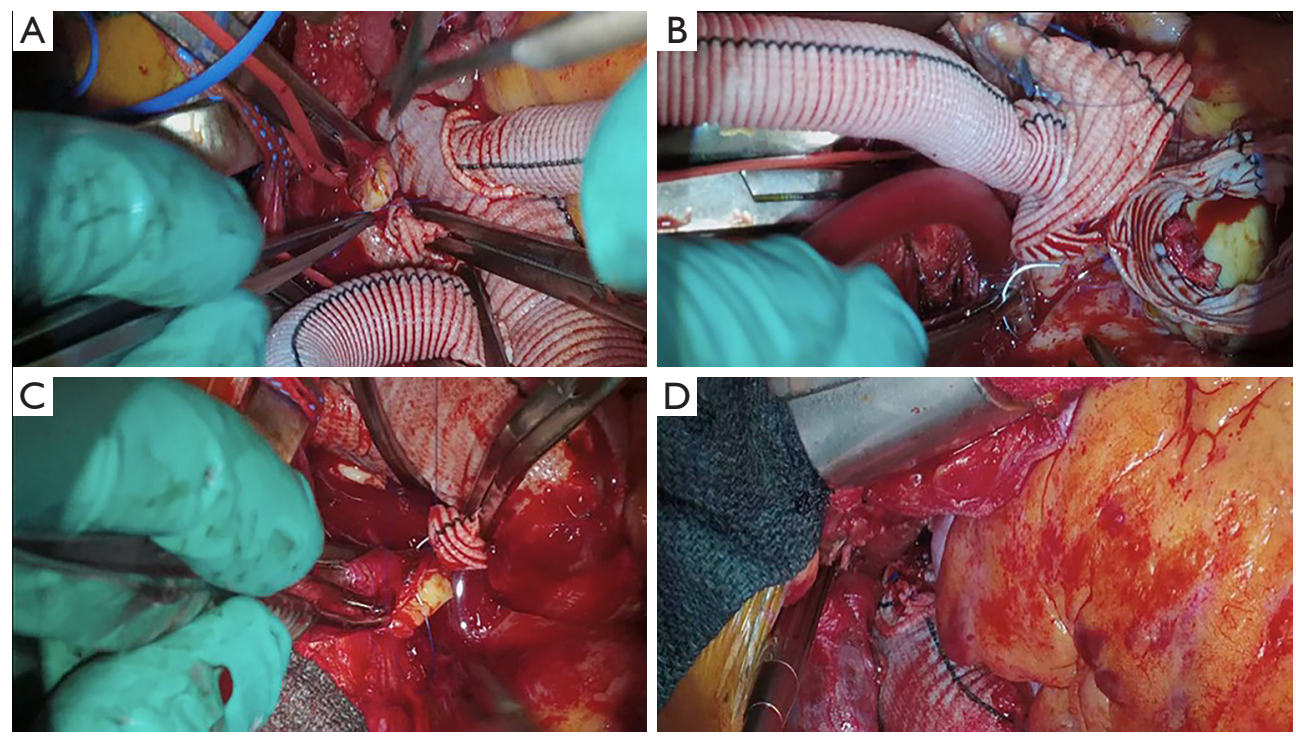

Figure 4 Surgical steps for arch and epiaortic vessel completion. (A) Cross-clamp of the ascending aorta, initiation of lower body reperfusion through the Thoraflex side limb, and construction of the carotid limb to the left common carotid artery; (B) anastomosis between the ascending aortic graft and the aortic arch graft; (C) removal of cross clamp, and construction of the innominate limb to the innominate artery; (D) final hemostatic result of the hybrid frozen elephant trunk aortic repair.

that the hybrid arch FET reconstruction was intact without any signs of pseudoaneurysm, graft kinking or any other significant abnormalities. The FET was well seated in the descending thoracic aorta and there were no signs of any endoleaks. At 3 months follow-up, the patient was found to be completely asymptomatic and was back to driving and enjoying his daily activities without any limitations. His echocardiogram and CT (Figure 5) scan at follow-up revealed excellent arch reconstructive results with widely patent epiaortic vessels and a well seated FET without complication.

\section{Comments}

We illustrate the steps for repair of an acute type A IMH involving the ascending aorta and arch using the Thoraflex Hybrid prosthesis. IMH falls within the spectrum of acute aortic syndromes, where definitive diagnoses can be somewhat 'grey', such as between IMH and penetrating aortic ulcer, as our demonstrated case. IMH is classically described as a contained aortic medial hematoma commonly believed to be secondary to intramural hemorrhage but without detectable intimal tear (11). Unlike classic aortic dissection, IMH has no mechanisms of decompression by a re-entry tear which places the patient at an unacceptable high risk of conversion into an acute aortic dissection and rupture (9). There is a general consensus that acute IMH involving the ascending aorta should be managed aggressively and surgically because of the unacceptably high mortality rates following medical treatment (12).

Since its introduction, the elephant trunk (ET) technique greatly facilitated the repair of extensive thoracic aortic disease with initial aortic arch reconstruction followed by a staged endovascular distal aortic repair. The ET procedure was designed with the aim of avoiding extensive dissection of scar tissue during the open distal aortic repair stage, which may jeopardize the left recurrent laryngeal and vagus nerves, the esophagus, and even the pulmonary artery. It also ensures short periods of hypothermic circulatory arrest by clamping the graft distal to the left subclavian artery rather than in the arch (13).

The antegrade deployment of thoracic endovascular aortic repair (TEVAR) stent grafts into the descending aorta opened yet a new chapter for the elephant trunk approach, allowing for a single-stage hybrid aortic arch repair strategy for treatment of complex aortic arch pathology, termed "frozen elephant trunk" (FET). The FET stent-graft deals with the problems of the short, "floppy," and radiolucent conventional Dacron ET that is often difficult to cannulate and can "accordion" on itself during endovascular repair. 

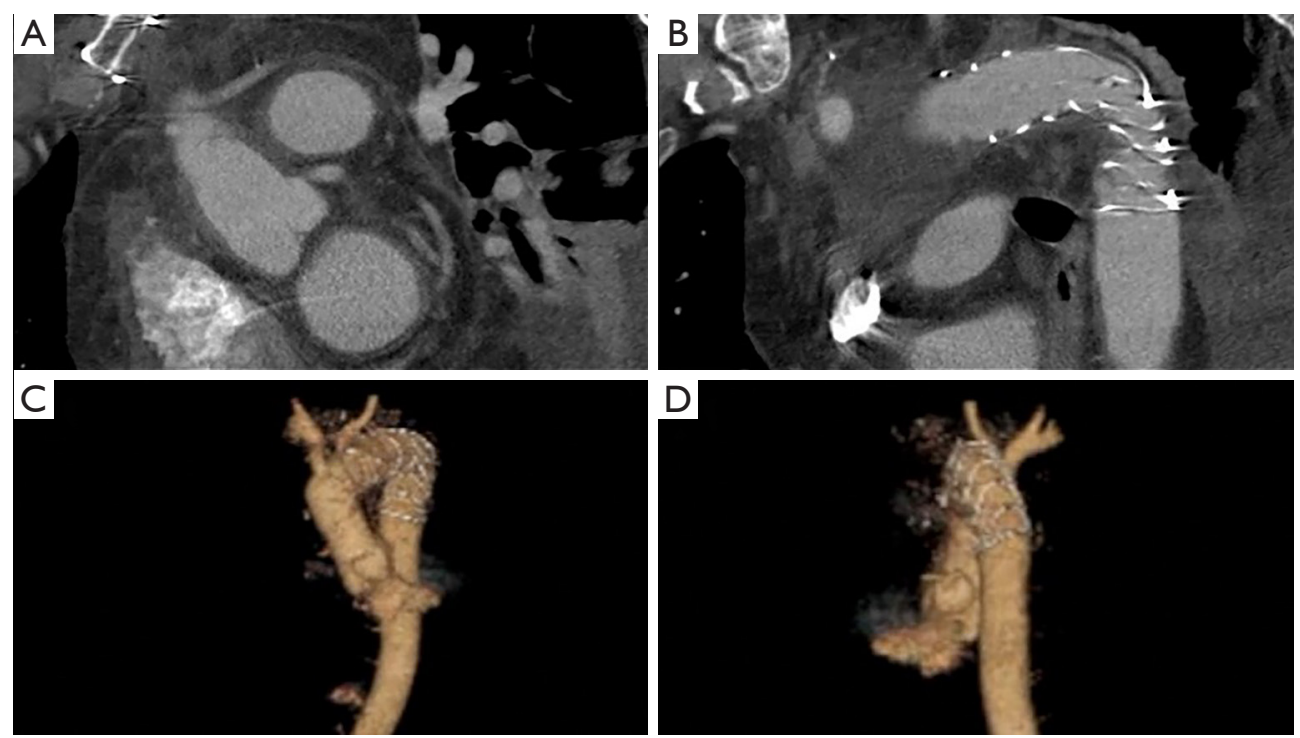

Figure 5 Three months follow-up computed tomography scan of the aorta with 3D reconstruction showing frozen elephant trunk reconstruction without any signs of pseudoaneurysm, graft kinking or endoleaks. The frozen elephant trunk was well seated in the descending thoracic aorta as seen on the $2 \mathrm{D}$ slices $(\mathrm{A}, \mathrm{B})$ and on the $3 \mathrm{D}$ reconstructions $(\mathrm{C}, \mathrm{D})$.

The FET technique also confers other advantages including the potential for false lumen thrombosis and aortic remodelling, which would be particularly useful in stabilizing complex aortic dissections or in our case an acute type A IMH with a fragile arch (2). In fact the FET technique has recently become our approach of choice in the treatment of extensive acute type A aortic dissection or IMH with complex anatomy.

However, the off-label use TEVAR stent-graft had multiple drawbacks. The blind antegrade deployment of the translucent TEVAR stent-graft often led to imprecision in the distal extent of aortic coverage, leading to deep implantation with higher risk of paraplegia or shallower implantation with inadequate distal seal and resultant type 1 endoleak (1). The TEVAR stent-grafts also pose greater technical challenges as they were never designed to accommodate the acute aortic angulations, were manufactured to be deployed at normothermia rather than during circulatory arrest or rewarming, and lacked side limbs.

Consequently, several single-piece endoprostheses were developed to further refine the FET repair. The commercially available hybrid arch grafts that are most commonly used are the Thoraflex Hybrid graft (Vascutek, Scotland), the E-vita Open Plus (Jotec, Germany), and the Frozenix J graft available in the Asian market. In principle, these commercially manufactured hybrid FET grafts have several potential benefits. In contrast to off label TEVAR stent-grafts, they are designed to be deployed antegrade and can be deployed without fluoroscopic guidance over a wire. Their design facilitates more proximal arch anastomosis in zone 1 or 2 which further simplifies the operation. They are also available in multiple lengths, which can be preselected to reduce the risk of paraplegia. Another graft prosthesis that has emerged is the Ascyrus Medical Dissection Stent (AMDS) which is an uncovered nitinol arch graft being used in the treatment of acute type A aortic dissection to promote acute true lumen expansion and enhance aortic remodeling (14). It is commonly used as an adjunct to a standard hemiarch anastomosis making for a much simpler operation; however, because if is uncovered, relies upon elimination of the primary entry tear and lack of distal re-entry tears.

The Thoraflex Hybrid prosthesis was introduced into the Canadian market in 2012 and consists of a fourbranched arch graft with a distal nitinol-ringed Dacron stent-graft. Three out of the four branches are anatomically oriented for epi-aortic vessel reconstruction with the remaining branch for earlier distal reperfusion. We opted to select the Thoraflex Hybrid graft because of its robust arch anastamotic collar, which can be particularly helpful in cases of friable, challenging to reach arch anastomosis 
with an acute aortic syndrome (15). The novel four-branch design of the Thoraflex prosthesis also enables arch first reconstruction using a distal arch collar (as opposed to head vessel first reconstruction strategies) and thus facilitates shorter lower body and myocardial ischemia time. Recent studies from the Canadian Thoracic Aortic Collaborative (CTAC) Investigators showed good early clinical results with low complication rates and excellent 1 - and 2-year patient outcomes associated with the Thoraflex Hybrid graft despite the early experiences with this device (5).

During our preoperative planning, we elected to perform a left carotid to subclavian bypass before aortic arch reconstruction to facilitate an easier arch repair because of the depth of the subclavian artery. In our experience, the decision about how to handle the left subclavian artery largely depends on the patient's anatomy on preoperative CT and urgency status (10). If the subclavian artery appears to be coursing deep and the patient is going for a non-emergent repair, then either a preoperative left carotid-subclavian bypass or transposition is performed. In emergent circumstances, primary intraoperative subclavian artery reconstruction directly to the subclavian limb of the Thoraflex is performed when anatomically feasible or extra-anatomic reconstruction of the subclavian to the left axillary artery becomes a valuable alternative. By following the outlined algorithm, we aim to decrease the timing of selective antegrade cerebral perfusion, cardiopulmonary bypass, and aortic cross-clamp times and the need for hypothermic circulatory arrest (16).

In summary, hybrid arch FET repair with a Thoraflex graft is a valuable technique that could be successfully applied to the management of extensive acute type A aortic dissection or IMH with complex anatomy.

\section{Acknowledgments}

None.

Footnote

Conflicts of Interest: MW Chu has received speaker's honorarium from Medtronic, LivaNova, Terumo Aortic, Abbott Vascular and Boston Scientific. The other authors have no conflicts of interest to declare.

\section{References}

1. Bozso SJ, White A, Nagendran J, et al. Hybrid aortic arch and frozen elephant trunk reconstruction: bridging the gap between conventional and total endovascular arch repair. Expert Rev Cardiovasc Ther 2018;16:209-17.

2. Hanif H, Dubois L, Ouzounian M, et al. Aortic arch reconstructive surgery with conventional techniques vs frozen elephant trunk: a systematic review and metaanalysis. Can J Cardiol 2018;34:262-73.

3. Shrestha M, Martens A, Krüger H, et al. Total aortic arch replacement with the elephant trunk technique: single-centre 30-year results. Eur J Cardiothorac Surg 2014;45:289-95; discussion 295-6.

4. Shrestha M, Kaufeld T, Beckmann E, et al. Total aortic arch replacement with a novel 4-branched frozen elephant trunk prosthesis: single-center results of the first 100 patients. J Thorac Cardiovasc Surg 2016;152:148159.e1.

5. Chu MW, Losenno KL, Dubois LA, et al. Early Clinical Outcomes of Hybrid Arch Frozen Elephant Trunk Repair with the Thoraflex Hybrid Graft. Ann Thorac Surg 2019;107:47-53.

6. Roselli EE. Trade in the hammer for a power driverperspectives on the frozen elephant trunk repair for aortic arch disease. Ann Cardiothorac Surg 2013;2:633.

7. Boufi M, Aouini F, Guivier-Curien C, et al. Examination of factors in type I endoleak development after thoracic endovascular repair. J Vasc Surg 2015;61:317-23.

8. Landau JH, Dubois LA, Chu MW. Hybrid arch frozen elephant trunk for persistent endoleak after thoracic endovascular aortic repair. Ann Thorac Surg 2017;104:e227-9.

9. Alomari IB, Hamirani YS, Madera G, et al. Aortic intramural hematoma and its complications. Circulation 2014;129:711-6.

10. Hage A, Ginty O, Power A, et al. Management of the difficult left subclavian artery during aortic arch repair. Ann Cardiothorac Surg 2018;7:414.

11. Coady MA, Rizzo JA, Elefteriades JA. Pathologic variants of thoracic aortic dissections: penetrating atherosclerotic ulcers and intramural hematomas. Cardiol Clin 1999;17:637-57.

12. Baikoussis NG, Apostolakis EE, Siminelakis SN, et al. Intramural haematoma of the thoracic aorta: who's to be alerted the cardiologist or the cardiac surgeon? J Cardiothorac Surg 2009;4:54.

13. Hagl C, Pichlmaier M, Khaladj N. Elephant trunks in aortic surgery: Fresh and frozen. J Thorac Cardiovasc Surg 2013;145:S98-102.

14. Bozso SJ, Nagendran J, MacArthur RG, et al. Dissected Aorta Repair Through Stent Implantation trial: Canadian 
results. J Thorac Cardiovasc Surg2019;157:1763-71.

15. Czerny M, Rylski B, Kari FA, et al. Technical details making aortic arch replacement a safe procedure using the Thoraflex ${ }^{\mathrm{TM}}$ Hybrid prosthesis. Eur J Cardiothorac Surg 2017;51:115-9.

Cite this article as: Hage F, Hage A, Chu MW. Hybrid arch frozen elephant trunk repair for acute type A intramural hematoma. Ann Cardiothorac Surg 2019;8(5):577-584. doi: 10.21037/acs.2019.08.01
16. Xydas S, Wei B, Takayama H, et al. Use of carotidsubclavian arterial bypass and thoracic endovascular aortic repair to minimize cerebral ischemia in total aortic arch reconstruction. J Thorac Cardiovasc Surg2010;139:717-22. 